\title{
Safety and efficacy of mini-percutaneous nephrolithotomy as daycare procedure: a prospective observational study
}

\author{
P. Aarthy, Mathisekaran Thangarasu* (1) J. Sanjay Prakash, Deepak Raghavan, Nitesh Jain, \\ Arunkumar Balakrishnan, M. Chandranathan, Sandeep Bafna, Rajesh Paul and Nivash Selvaraj
}

\begin{abstract}
Background: To evaluate the safety, feasibility of mini-percutaneous nephrolithotomy ( $\mathrm{mPCNL}$ ) being carried out as a daycare surgery and to study the re-admission, stone clearance rate and complication rates following mPCNL. We also sought to find out the rate of requirement of ancillary procedure, after $\mathrm{mPCNL}$.

Methods: In this prospective observational study, easily accessible patients above 18 years of age with renal and/or upper ureteric calculi, who underwent mPCNL between September 2018 and February 2020, were included. Seventy patients were selected, as per statistical methods. Preoperative evaluation including history, blood and urine investigations and radiological images was collected. Intra-operative and post-operative events were analyzed. Written consent was obtained from the patients to participate in the study and to publish their data. Institutional Ethical and scientific committee clearance was obtained.

Results: Out of 70 patients who underwent mPCNL in the study, in the age group ranging from 24-68 years, most of the cases were partial staghorn with maximum size of stone up to about $3.5 \mathrm{~cm}$. Inferior calyx was most frequently punctured. Six cases had multipuncture mPCNL, four patients had bilateral procedure, and two had supra-costal puncture. Eighteen patients had tubeless $\mathrm{mPCNL}$, of which four were totally tubeless procedures. Four patients had hematuria, none requiring transfusion. Two patients had sepsis, managed with higher antibiotics. Limitation of the study was the sample size. To derive a sturdy conclusion, large scale studies are recommended.

Conclusions: We can conclude that $\mathrm{MPCNL}$ can be safely done as daycare or ambulatory surgery in properly selected patients. In centers with experienced urologists, bilateral and multipuncture MPCNL can be done as an ambulatory surgery. Thus, this addresses the stone disease as well as cost containment, without patient safety being compromised. Bed occupancy rate is reduced, helping effective utilization of hospital resources.
\end{abstract}

Keywords: Percutaneous access, Kidney calculi, Ureteral calculi, Daycare

\section{Background}

High prevalence, increasing incidence combined with a high recurrence rate of urolithiasis, makes the ideal treatment modality and goal to be elusive $[1,2]$. Treatment aims at complete clearance of calculi without any residual fragments, along with pain management and complete

\footnotetext{
*Correspondence: mathisekaran@gmail.com

Department of Genitourinary Surgery, Apollo Main Hospital, 21, Gream's Lane, Off Gream's Road, Chennai 600006, India
}

eradication of the causative micro-organisms [3]. Percutaneous nephrolithotomy (PCNL) is now being offered as a standard treatment option for patients with complex and fairly large renal stones. Further improvisation of instruments and techniques has resulted in "miniperc" and "ultraperc," which were found to be safe and effective [4]. The aim of the present study is to evaluate the safety and feasibility of mPCNL, being carried out as an ambulatory surgery. The objectives are to identify the readmission rate, stone clearance and the complication rate 
following daycare $\mathrm{mPCNL}$, where the patient gets operated, recovers and returns home within a day or within $24 \mathrm{hrs}$ of the surgery [5].

\section{Methods}

This study is a prospective observational study which was conducted in our hospital, Department of Urology, between September 2018 and February 2020. The target population were the patients who underwent mPCNL as a daycare surgery. The sample size is 70 in our study.

Inclusion Criteria include that all patients above 18 years with renal and/or upper ureteric calculi and who underwent mPCNL.

Exclusion Criteria were patients who were found to have evidence of active infection in urine, altered renal anatomy such as pelvic kidney, co-morbidities that preclude the surgery (coagulation abnormalities or cardiac, respiratory and other co-morbidities) and complete staghorn calculus.

All the patients who underwent mini-percutaneous nephrolithotomy (mPCNL) in the specified time period were included as per the inclusion \& exclusion criteria, after explaining about the study and getting written, informed consent from the patient for participating in the study and publishing the data. Institutional scientific and ethical committee clearance was obtained for the study. The procedures adhered to the ethical guidelines of Declaration of Helsinki and its amendments.

Pre-operative Evaluation included detailed medical history, physical examination and hematological investigations. Patients with positive urine cultures were given intravenous antibiotics pre-operatively for 5 days. Intravenous Urogram (IVU) or computerized tomography (CT) to assess stone size, site, anatomy of the pelvicalyceal system was done to plan optimal access to the renal calculi. The size of the stone was measured by analyzing the stones' longest diameter or in cases of multiple calculi by measuring the sum of each stones' diameter. All the patients were operated under general anesthesia. In supine-mPCNL, patient was placed in Galdakao-modified Valdivia position. In prone-mPCNL patient was placed in prone position with supporting rolls below the shoulder and pelvis. Percutaneous access to the Pelvicalyceal system (PCS) was performed under fluoroscopic guidance. Mini-PCN sheath and a 12Fr nephroscope were used. Stone fragmentation was done using pneumatic lithoclast or Holmium laser. Irrigation, flushing or grasper were used to extract small stones. Stone clearance was confirmed by endoscopy and fluoroscopy. Double J(DJ stent) or ureteric catheter was placed postsurgery, based on surgeon preference. Nephrostomy was deployed in cases of renal pelvis injury, abnormal calyceal anatomy and calyceal diverticular calculi. Time from starting of cystoscopy to withdrawal of Amplatz sheath was taken as operative time. None of the patients included in the study underwent endoscopic combined intra-renal surgery. The PCN tract was infiltrated with anesthetic agent $(0.25 \%$ bupivacaine) once the procedure was completed. Intra-operative complications were recorded.

Post-operatively patients were observed for minimum of $6 \mathrm{~h}$ to look for complications such as hematuria, sepsis, pain based on Visual Analog Score(VAS), signs of other complications and vitals were monitored. Persistent hematuria, hypotension or other complications were a markers for re-admission and further observation and management. Patient was discharged only if they progressed well. Outpatient follow-up was done on post-operative day (POD) -1. The nephrostomy tube and Foley's catheter (the following day if no urine leak) were removed in cases where they were deployed. Foley's catheter and retrograde ureteric catheter (if deployed) were removed on POD-1, if there was no nephrostomy tube. On POD-5, patients were reviewed with serum creatinine, hemoglobin and X-ray of the KUB region (or CT of radiolucent calculi). In case of residual stone fragments, ancillary procedure was done. DJ stents, if deployed, were removed 6-8 weeks after surgery. Complications were graded as per modified Clavien-Dindo classification.

\subsection{Statistical methods}

Continuous and categorical variables were expressed as mean \pm standard deviation and percentages, respectively. Comparisons of categorical variables and continuous variables were done by Chi-square test and independent t-test, respectively. p-values less than 0.05 were considered statistically significant. Data analysis was achieved by using SPSS software version 16.0.

\section{Results}

The demographic data of the patients are discussed in Table 1.

The most common complaint is flank pain (65.7\%), and $17 \%$ of patients had their stones incidentally

Table 1 Parameters of patients undergoing mPCNL

\begin{tabular}{llllll}
\hline Parameters & $\boldsymbol{N}$ & Minimum & Maximum & Mean & SD \\
\hline Descriptive statistics & & & & & \\
Age & 70 & 24 & 68 & 45.06 & 10.671 \\
BMI & 70 & 18.1 & 33.2 & 24.820 & 4.0240 \\
Pre-op hemoglobin & 70 & 7.5 & 16.7 & 12.766 & 2.0716 \\
Pre-op Creatinine & 70 & 0.5 & 7.10 & 1.2546 & 1.26149 \\
Size of stone in cm & 70 & 2.0 & 3.5 & 2.480 & 0.4862 \\
\hline
\end{tabular}


Table 2 Site of renal calculi in Patients

\begin{tabular}{llc}
\hline Site of stone & Frequency $(\boldsymbol{n})$ & Percent $(\%)$ \\
\hline Inferior calyx & 10 & 13.6 \\
Middle calyx & 6 & 8.1 \\
Upper calyx & 4 & 5.4 \\
Pelvis & 22 & 29.7 \\
Partial staghorn & 24 & 32.4 \\
Proximal ureter & 4 & 5.4 \\
Inferior calyx + middle calyx & 2 & 2.7 \\
Inferior calyx + upper calyx & 2 & 2.7 \\
Total renal units & 74 & 100.0 \\
\hline
\end{tabular}

Table 3 Intraoperative parameters of patients undergoing MPCNL

\begin{tabular}{|c|c|c|c|c|}
\hline Parameters & Minimum & Maximum & Mean & SD \\
\hline \multicolumn{5}{|l|}{ Descriptive statistics } \\
\hline $\begin{array}{l}\text { Duration of surgery in } \\
\text { mins }\end{array}$ & 50.00 & 120.00 & 80.0 & 19.70369 \\
\hline Hospital Stay in hours & 7 & 60 & 17.25 & 11.23 \\
\hline Post-op Hb & 7.10 & 15.90 & 12.4571 & 1.90484 \\
\hline Post-op serum creatinine & 0.60 & 5.40 & 1.1057 & 0.86158 \\
\hline
\end{tabular}

detected. Out of 70 patients, 14 patients (20\%) were found to be diabetic and 28 patients (40\%) were hypertensive. Right-sided calculi were seen in 42 (60.0\%) patients and left side calculi in 24 patients $(34.3 \%)$ and bilateral in about $4(5.7 \%)$ patients. Table 2 shows the sites of stone lodging.

Two patients had stone in upper and lower calyx, and two others had in middle and lower calyx. The average stone size was $2.48 \mathrm{~cm}$ (range $2.0-3.5 \mathrm{~cm}$ ). Table 3 shows intraoperative and postoperative parameters.

The change in $\mathrm{Hb}$ level was found to be statistically significant, but clinically it was not significant. The significant change in creatinine value was mainly due to fall of creatinine values in patients with azotemia, due to the relief of obstruction and hence resolving acute kidney injury (Table 4).
All 70 patients had radio-opaque calculi, 60 patients (85.7\%) underwent prone $\mathrm{mPCNL}$, and $10(14.3 \%)$ of patients underwent supine $\mathrm{mPCNL}$. The most common site of puncture was inferior calyx (70.3\%), followed by middle (16.2\%) and upper calyx (5.4\%). In six renal units, dual punctures were done into the middle and lower calyx.

Only two patients had supracostal puncture (supra twelfth) into the upper calyx, and all other patients had infracostal puncture. Complete stone clearance was achieved in $91.4 \%$ of patients. In $88.6 \%$ of patients $(n=62)$, DJ stenting was done and in $11.4 \%(n=8)$ RGC was left in situ. PCN was deployed following PCNL in 18 cases $(25.7 \%)$ and not in the rest of the 52 patients. No intra-operative complications were seen in any of the patients. About $68.6 \%$ of patients had mild pain, Visual Analogue Score (VAS) [one, two], 17.1\% of patients, experienced moderate pain VAS-[three, four], 14.3\% experienced no pain and none of them had VAS more than 4.

Only six patients were admitted in the ward. The rest were discharged as daycare patients. Two patients (2.9\%) had signs of sepsis (fever with rigors, tachycardia and hypotension) and were treated with fluids and higher antibiotic (Meropenem $1 \mathrm{~g}$ ), and they responded well (Clavien-Dindo grade 2). Four patients had hematuria (Clavien-Dindo grade 1), responded well to intravenous fluid and diuretic (furosemide). No blood transfusion was required. There was no complication in $91.4 \%$ patients. In $5.7 \%$ patients, there was hematuria not warranting blood transfusion. In $8.6 \%$ of patients, ancillary procedureShockwave lithotripsy (SWL), was done and in the rest complete stone clearance was achieved.

\section{Discussion}

Renal calculi result from disturbance in the balance between precipitation and solubility of salts with most calculi containing calcium [6]. At least $10 \%$ of people are affected by Urolithiasis. Almost $70 \%$ of people who suffer kidney stone will have recurrence [7]. In Asia, between 1 and 19.1\% suffer from urolithiasis. Recurrence rate of renal calculi varies in between 21 and 53\% after $3-5$ years. In a study by Kruck et al, the recurrence rates

Table 4 Hemoglobin and creatinine levels of patient before and after surgery

\begin{tabular}{lllllll}
\hline & Parameters & Mean & N & SD & Std. Error mean & P value \\
\hline Pair 1 & Pre-op Hb & 12.766 & 35 & 2.0716 & 0.3502 & 0.0001 \\
& Post-op Hb & 12.4571 & 35 & 1.90484 & 0.32198 & 0.21323 \\
Pair 2 & Pre-op Creatinine & 1.2546 & 35 & 1.26149 & 0.060 \\
& Post-op serum creatinine & 1.1057 & 35 & 0.86158 & 0.14563 & \\
\hline
\end{tabular}


were found to be much lesser when comparing $\mathrm{mPCNL}$ with retrograde intrarenal surgery [8].

The mean body mass index (BMI) of patients in our study was $24.82 \pm 4.04 \mathrm{~kg} / \mathrm{m}^{2}(18.1-33.2)$ which was almost similar to the study by Walid Sharour et al. where the mean BMI of the patients was $25.9 \mathrm{~kg} / \mathrm{m}^{2}(22.9-31.7)$ [9]. BMI is important, mainly because of the skin-stone distance requiring longer sheaths and positioning of the patient. All patients were stable clinically with no requirement of blood transfusions. To prevent recurrence and to preserve renal function, it is crucial to correct any underlying metabolic or anatomical abnormalities $[10,11]$.

Surgical treatment has drifted from open surgeries to endoscopic surgeries [1]. Recent developments in PCNL and SWL have made management better [12]. PCNL is minimally invasive procedure for removal of renal stones that are more than two centimeters in size. In 1976, Fernstorm and Johanssen, for the first time, reported the removal of renal stones through a nephrostomy tract [13]. PCNL has been identified globally for its safety with considerable success rate and reduced complications suitable for all age groups including pediatric cases [14, 15]. It facilitates a direct approach to the calculus and subjects surrounding structures to less trauma. Many modifications have come up, including use of regional blocks, single-step dilatation, "Mini-Perc" technique and ultraperc(are associated with lesser frequency and severity of complications) [4], tubeless PCNL and sandwich therapy $[12,16]$. mPCNL has been promoted as an alternative monotherapy for staghorn and multiple renal stones [17]. However, experience of the urologist also plays a crucial role. Most available reports are from developed countries, and there is shortage of data from developing and underdeveloped countries [18]. Most institutes in India still practice $\mathrm{mPCNL}$ as an in-patient procedure.

In our study, most of the stones were partial staghorn $32.4 \%$. In two patients, stone was there in upper and lower calyx and in two others, stone was in middle and inferior calyx. In the study done by El-Tabey et al., most of the stones were located in the renal pelvis $-50 \%(42)$, followed by inferior calyx-28.6\% (24) and then partial staghorn-21.4\% [19].

Walid Sharour et al's single-puncture PCNL study demonstrated a $70 \%$ inferior, $20 \%$ middle and 10\% upper calyceal puncture which is similar to our study [9]. Desai et al. also advocate the use of mPCNL for a safer anterior calyx and supra-costal puncture [20]. In our study, 18 patients underwent $\mathrm{mPCNL}$ without $\mathrm{PCN}$ insertion, amongst whom, stenting was also not done in 4 patients (5.7\%)-completely tubeless PCNL. In Ahmed Fahmy et al. study, 128 patients (79\%) underwent tubeless PCNL and 34 patients (21\%) had PCN deployed, which is almost similar to our study [21].

The operative time was calculated from the beginning of cystoscopy to the withdrawal of sheath. The average operative time in our study was $80 \pm 19.7 \mathrm{~min}$ (range of 50-120 min). The mean operative time was similar to the studies conducted by Fahmy et al. (94 min), Sharma et al. (72 min), Beiko et al. (86 min), Walid et al. (83.5 min) $[9,21,22,23]$. It was comparatively higher than the studies conducted by Iqbal Singh et al. (48.8 $\mathrm{min}$ ) and El-Tabey et al. $(46.6 \pm 6.3 \mathrm{~min})[19,24]$. This higher mean operative time could be secondary to lengthier operative time taken for 4 cases of bilateral PCNL and 6 units of multipuncture PCNL.

The mean hospital stay in our study was $17.25 \pm 11.23 \mathrm{~h}$ with the earliest discharge at $7 \mathrm{~h}$ and maximum being $60 \mathrm{~h}$. The mean hospital stay in El-Tabey et al. and Iqbal Singh et al. studies was $33.4 \pm 17.5 \mathrm{~h}(24-96 \mathrm{~h})$ and $40 \mathrm{~h}$ which showed longer hospital stay than our study [19, 24]. In Sharma et al. (12.5 h, range 5.5-23.5) and Ahmed et al. $(9 \mathrm{~h})$, the average hospital stay was shorter as they excluded the duration of stay of the re-admitted patients and calculated the average from the patients discharged as day-care $[21,22]$.

In our study, $91.4 \%$ of patients were completely cleared off the stone, which was similar to studies by Ahmed Fahmy et al. (SFR- 90.7\%) and El Taby et al. (SFR- 91.7\%) $[19,21]$. In studies by Iqbal Singh et al., and Sharma et al., $100 \%$ stone clearance was achieved $[22,24]$.

The readmission rate is $8.6 \%(n=6)$, similar to the studies by Fahmy et al., (9.8\%) [21] and Sharma et al. (15\%) [22], and it is better than El-Tabey et al. study (28.6\%) [19]. Two patients (2.9\%) in our study had sepsis and were treated with higher antibiotic (intravenous Inj.Meropenem $1 \mathrm{~g}$ ) and responded effectively. Six of $162(3.7 \%)$ patients in Fahmy et al. study [21], one of $50(2 \%)$ patients in Beiko et al. study [23], five of 84 patients (6\%) of ElTabey et al. study [19] and one of 10 patients (10\%) of WalidSharour et al. study [9] had signs of sepsis requiring admission and higher antibiotics. The emergency department visit and readmission rates of ambulatory PCNL in a study done by Schoenfeld et al. was 11\% [25]. Bechis et al., in their study, found that the urologist performing daycare $\mathrm{mPCNL}$ should be prepared to admit $10 \%$ of well selected daycare $\mathrm{MPCNL}$ patients and approximately $18 \%$ of non-selected patients after the procedure [26].

None of the patients in our study had colonic injury or hydrothorax. Six patients (8.6\%) in our study had residual calculi and underwent SWL as an ancillary procedure before removing the stent, which was higher than the El-Tabey et al. study (4.8\%). There is continuous miniaturization of the PCNL techniques, like micro-PCNL, 
mini-micro-PCNL and super-mini-PCNL. The supermini-PCNL technique has lesser complication rate when compared to $\mathrm{mPCNL}$ as well as better stone clearance rates in pediatric patients [27].

\subsection{Limitation}

Sample size was as per statistical calculation but to derive a sturdy conclusion large scale studies are recommended. However, this has been done as a pilot study and more cases of mPCNL as well as PCNL are being done as a daycare surgery and the data are being collected which could provide valuable changes in the near future.

\section{Conclusion}

Out of 70 patients, most of the cases were partial staghorn with maximum stone size upto $3.5 \mathrm{~cm}$, inferior calyx was the most frequently punctured with $6 \mathrm{mul}-$ tipuncture mPCNL. Four patients had bilateral procedure with 2 supracostal puncture. Eighteen patients had tubeless $\mathrm{mPCNL}$, of which four were totally tubeless procedures. No major intraoperative or postoperative complications were noted. We can conclude that in tertiary care centers with experienced urologists and in properly selected patients, bilateral as well as multipuncture $\mathrm{mPCNL}$ can be safely done as a daycare surgery. Faster recovery, reduced bed occupancy and cost-effective care for patients can thus be obtained. Since experienced urologists performed all cases, those surgeons in the earlier part of learning curve of the procedure have to decide carefully before discharging the multipuncture, supracostal puncture or bilateral PCNL patients as day-care.

\section{Abbreviations \\ mPCNL: Mini-percutaneous nephrolithotomy; IVU: Intravenous urogram; CT: Computerized tomography; PCS: Pelvicalyceal system; DJ: Double J; VAS: Visual analog score; POD: Post-operative day; RGC: Retrograde ureteric catheter; SWL: Shockwave lithotripsy; BMI: Body mass index; PCN: Percutaneous nephrostomy.}

\section{Acknowledgements}

We are duty bound to acknowledge the support of our patients for allowing us to conduct the study, our Biostatistician Mr. Balasubramaniam for his contribution in statistical analysis and the health care workers who supported in the entire journey.

\section{Authors' contributions}

AP, MT, DR, NJ and AKB done Concepts, Design, Definition of intellectual content, Literature search, Clinical studies, Experimental studies, Data acquisition, Data analysis, Statistical analysis, Manuscript preparation, Manuscript editing and Manuscript review. SPJ and SB done Design, Definition of intellectual content, Literature search, Clinical studies, Experimental studies, Data acquisition, Data analysis, Statistical analysis, Manuscript preparation, Manuscript editing and Manuscript review. CM done Concepts, Design, Definition of intellectual content, Literature search, Experimental studies, Data acquisition, Data analysis, Statistical analysis, Manuscript preparation, Manuscript editing and Manuscript review. RP and NS done Design, Definition of intellectual content, Literature search, Experimental studies, Data acquisition, Data analysis, Statistical analysis, Manuscript preparation, Manuscript editing and Manuscript review. All the authors have contributed in few surgeries each and case management, concept and design of the study, data collection, analysis and validation, writing of the literature, manuscript editing and review. All authors have read and approved the manuscript and agreed for authorship criteria set, by the journal editorial committee. Guarantor for the above statement would be the corresponding author.

\section{Availability of data and material}

Available on reasonable request from the corresponding author.

\section{Consent for publication}

Written consent was obtained from participants of the study.

\section{Ethics approval and consent to participate}

Name of the committee which gave ethical clearance for study: Institutional Ethics Committee-Clinical studies, Apollo Hospitals, 1/16 \&1/17, 2nd Floor, Krishna Deep Chambers, Wallace Garden Street, Chennai-600 006. All the patients who underwent mini-percutaneous nephrolithotomy (mPCNL) in the specified time period were included as per the inclusion \& exclusion criteria, after explaining about the study and getting written, informed consent from the patient for participating in the study and publishing the data.

\section{Competing interests}

The authors declare that they have no competing interests.

Received: 14 July 2020 Accepted: 28 December 2020

Published online: 09 January 2021

\section{References}

1. Holman E, Khan AM, Flasko T, Toth C, Salah MA (2004) Endoscopic management of pediatric urolithiasis in a developing country. Urology 63(1):159-162

2. Salerno A, Nappo SG, Matarazzo E, De Dominicis M, Caione P (2013) Treatment of pediatric renal stones in a Western country: a changing pattern. J Pediatr Surg 48(4):835-839

3. Onal B, Citgez S, Tansu N, Emin G, Demirkesen O, Talat Z, Yalcin V, Erozenci A (2013) What changed in the management of pediatric stones after the introduction of minimally invasive procedures? A single-center experience over 24 years. J Pediatr Urol 9(6):910-914

4. Desai MR, Kukreja RA, Patel SH, Bapat SD (2004) Percutaneous nephrolithotomy for complex pediatric renal calculus disease. J Endourol 18(1):23-27

5. Preminger GM, Clayman RV, Curry T, Redman HC, Peters PC (1986) Outpatient percutaneous nephrostolithotomy. J Urol 136(2):355-357

6. Han H, Segal AM, Seifter JL, Dwyer JT (2015) Nutritional management of kidney stones (nephrolithiasis). Clin Nutr Res 4(3):137-152

7. Moe OW (2006) Kidney stones: pathophysiology and medical management. Lancet 367(9507):333-344

8. Kruck S, Anastasiadis AG, Herrmann TR, Walcher U, Abdelhafez MF, Nicklas AP, Hölzle L, Schilling D, Bedke J, Stenzl A, Nagele U (2013) Minimally invasive percutaneous nephrolithotomy: an alternative to retrograde intrarenal surgery and shockwave lithotripsy. World J Urol 31(6):1555-1561

9. Shahrour W, Andonian S (2010) Ambulatory percutaneous nephrolithotomy: initial series. Urology 76(6):1288-1292

10. Frattini A, Ferretti S, Salvaggio A (2010) Percutaneous nephrolithotripsy (PCNL) in children: experience of Parma. Arch Ital Urol Androl 82(1):51-52

11. Liu B, Wu Y, Wei J, Gao J (2008) Treatment of pediatric renal calculi between 1990 and 2006 in Henam province. Life Sci J 2008:525-529

12. Saied MM, Sonbul ZM, El-Kenawy M, Atallah MM (1991) Spinal and interpleural bupivacaine for percutaneous nephrolithotomy. Middle East J Anaesthesiol 11(3):259-264

13. Fernström I, Johansson B (1976) Percutaneous pyelolithotomy: a new extraction technique. Scand J Urol Nephrol 10(3):257-259

14. Mor Y, Elmasry YE, Kellett MJ, Duffy PG (1997) The role of percutaneous nephrolithotomy in the management of pediatric renal calculi. J Urol 158(3):1319-1321

15. Nasirov FR, DKh M, Shl G, Alidjanov JF, Abdufattaev UA, ZhM Y, Mukhtarov S, Akilov FA (2015) Evaluation of the efficacy of standard percutaneous 
nephrolithotripsy in staghorn and multiple nephrolithiasis. Urologiia (Moscow, Russia: 1999) 1(1):66-69

16. Jackman SV, Hedican SP, Peters CA, Docimo SG (1998) Percutaneous nephrolithotomy in infants and preschool age children: experience with a new technique. Urology 52(4):697-701

17. Unsal A, Resorlu B, Kara C, Bozkurt OF, Ozyuvali E (2010) Safety and efficacy of percutaneous nephrolithotomy in infants, preschool age, and older children with different sizes of instruments. Urology 76(1):247-252

18. Samad L, Aquil S, Zaidi Z (2006) Paediatric percutaneous nephrolithotomy: setting new frontiers. BJU Int 97(2):359-363

19. El-Tabey MA, Abd-Allah OA, Ahmed AS, El-Barky EM, Noureldin YA (2013) Preliminary study of percutaneous nephrolithotomy on an ambulatory basis. Curr Urol 7(3):117-121

20. Desai J, Zeng G, Zhao Z, Zhong W, Chen W, Wu W (2013) A novel technique of ultra-mini-percutaneous nephrolithotomy: introduction and an initial experience for treatment of upper urinary calculi less than $2 \mathrm{~cm}$. Biomed Res Int 1:2013

21. Fahmy A, Rhashad H, Algebaly O, Sameh W (2017) Can percutaneous nephrolithotomy be performed as an outpatient procedure? Arab J Urol 15(1):1-6
22. Sharma AK, Nagabhushan M, Girish GN, Kamath AJ, Ratkal CS, Venkatesh GK (2013) Analysis of the feasibility and efficacy of ambulatory/day care percutaneous nephrolithotomy: an initial experience. UroToday Int J 6:44

23. Beiko D, Samant M, McGregor TB (2009) Totally tubeless outpatient percutaneous nephrolithotomy: initial case report. Adv Urol 1:2009

24. Singh I, Kumar A, Kumar P (2005) "Ambulatory PCNL" (tubeless PCNL under regional anesthesia)-A preliminary report of 10 cases. Int Urol Nephrol 37(1):35-37

25. Schoenfeld D, Zhou T, Stern JM (2019) Outcomes for patients undergoing ambulatory percutaneous nephrolithotomy. J Endourol 33(3):189-193

26. Bechis SK, Han DS, Abbott JE, Holst DD, Alagh A, DiPina T, Sur RL (2018) Outpatient percutaneous nephrolithotomy: the UC San Diego health experience. J Endourol 32(5):394-401

27. Zeng G, Zhu W, Lam W (2018) Miniaturised percutaneous nephrolithotomy: its role in the treatment of urolithiasis and our experience. Asian J Urol 5(4):295-302

\section{Submit your manuscript to a SpringerOpen ${ }^{\odot}$ journal and benefit from:}

- Convenient online submission

- Rigorous peer review

- Open access: articles freely available online

- High visibility within the field

- Retaining the copyright to your article

Submit your next manuscript at $\boldsymbol{\nabla}$ springeropen.com 